\title{
ARTIGO
}

\section{APLICAÇÃO DO MÉTODO TAGUCHI NA OTIMIZAÇÃO DOS PARÂMETROS DA OPERAÇÃO DE FURAÇÃO DE UM MATERIAL SANDUÍCHE ${ }^{1}$}

\author{
Bruna Aparecida Rezende ${ }^{2}$ \\ Michele Lisboa Silveira \\ Juan Carlos Campos Rubio
}

\section{RESUMO}

Concomitantemente à necessidade de desenvolvimento de materiais com qualidades superiores surge a necessidade de processar estes novos materiais, apresentando características e/ou dificuldades para a fabricação de componentes. Neste sentido, os materiais compósitos apresentam diversas características, como baixo peso, rigidez e resistência elevada, que permitem o uso em diversas áreas da indústria moderna. Os materiais sanduíche são um tipo de compósito estrutural e apresentam basicamente 2 faces e um núcleo. Para se alcançar produtos com as qualidades desejadas, são utilizados diferentes processos de fabricação, dentre eles a usinagem. A operação de furação é uma das mais utilizadas para a junção de materiais. Diversos problemas podem decorrer da mesma, como por exemplo, delaminação, erro de circularidade e a presença de rebarbas. Neste sentido, o presente trabalho teve por objetivo a determinação das condições ótimas de corte, por meio do Método Taguchi, para a furação de um material tipo sanduíche, constituído de alumínio e polietileno. Foram utilizados três tipos de ferramentas, três velocidades de corte e três avanços. As condições de corte ótimas obtidas foram estabelecidas considerando a menor força de avanço, menor altura da rebarba e menor diâmetro máximo.

Palavras-chave: Furação. Material Sanduíche. Método Taguchi.

\section{INTRODUÇÃO}

A maioria dos produtos modernos exige materiais com características diferenciadas, as quais são possíveis por meio da combinação de polímeros, cerâmicas e metais, visto que as propriedades desejadas não podem ser encontradas nestas classes de materiais separadamente. Dentre estes, têm-se os materiais sanduíches, que buscam apresentar baixo peso, boa resistência ao impacto e rigidez.

\footnotetext{
${ }^{1}$ Como citar este artigo: REZENDE, Bruna Aparecida; SILVEIRA, Michele Lisboa; RUBIO, Juan Carlos Campos. Aplicação do método Taguchi na otimização dos parâmetros da operação de furação de um material sanduíche. ForScience: revista científica do IFMG, Formiga, v. 6, n. 2, p. 00402, 2018. out. Edição especial.
}

${ }^{2}$ Autor para correspondência: Bruna Aparecida Rezende, IFMG - Campus Bambuí, bruna.rezende@ifmg.edu.br. 
O material sanduíche utilizado neste trabalho é constituído de alumínio e polietileno e possui diversas aplicações, como em revestimento de comércios, faixas, transportes rodoviários, indústria aeronáutica e naval, entre outras.

A operação de furação é uma das mais utilizadas na indústria devido à necessidade de efetuar montagens por meio da junção mecânica com parafusos e rebites, como, por exemplo, nas aplicações aeroespaciais (KHORAN et al., 2015). Para aumentar a produtividade e consequentemente maximizar o lucro, é necessária a utilização de parâmetros corretos de usinagem, pois assim podem ser minimizados os problemas. Entre os problemas comuns, oriundos da furação de materiais sanduíche, podem ser citados a delaminação, a presença de rebarba e os desvios dimensionais. Os defeitos nos furos representam um alto percentual de rejeição na operação de furação, sendo estes responsáveis por cerca de $60 \%$ na rejeição das peças, como afirmam Zitoune, Krishnaraj e Collombet (2010).

Durante a furação, a ferramenta (broca) gira realizando movimento de corte em conjunto com o movimento de avanço (penetração) e faz a retirada contínua de material (MACHADO et al., 2015). Durante a furação, uma broca helicoidal está sujeita aos esforços de torção e compressão. A torção se deve ao fato de que, à medida que a ferramenta avança, é realizado o movimento de rotação e a compressão é decorrente deste avanço. Portanto, para se mensurar os esforços na furação, basta calcular o momento torsor e a força de avanço (DINIZ; MARCONDES; COPPINI, 2006). As forças de usinagem, na furação, podem ser decompostas em basicamente três: Força de corte $(\mathrm{Fc})$, Força de avanço $(\mathrm{Ff})$ e Força passiva (Fp), apresentadas na figura 1:
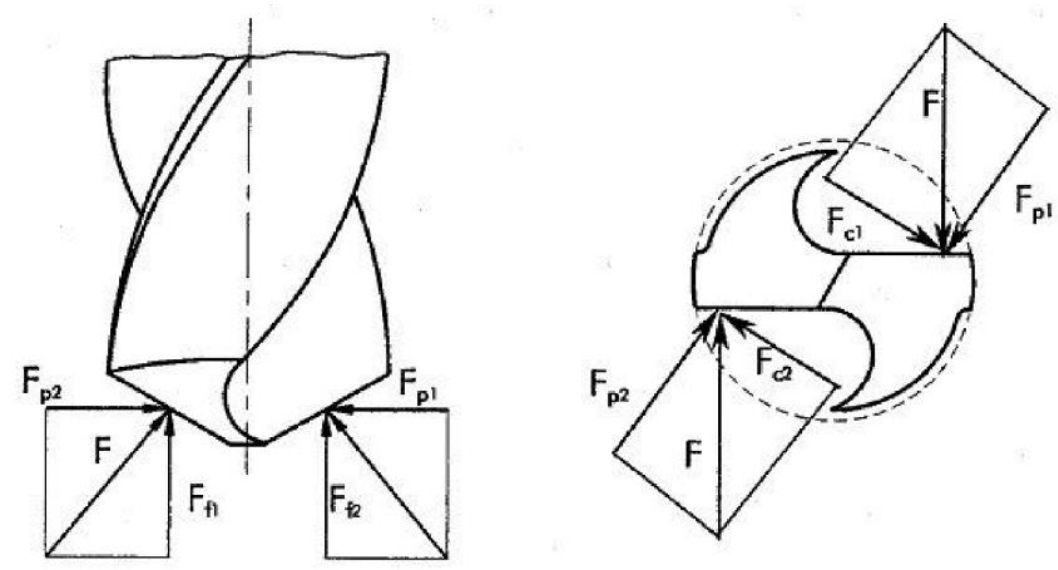

Figura 1 - Forças atuantes nas arestas principais de corte Fonte: Stemmer (2005 apud OLIVEIRA, 2008, p. 40).

Fatores importantes a serem analisados neste estudo de furação são a rebarba na saída do furo e a circularidade. A eliminação da rebarba representa um custo de $30 \%$ na operação 
de furação sendo esta decorrente da deformação plástica do material da peça e pode ser formada na entrada ou na saída do furo (PILNÝ et al., 2012). Por outro lado, a circularidade indica o quanto um círculo pode destoar de um círculo perfeito. Essas variáveis respostas apresentam influência direta na operação de furação, pois podem comprometer o produto final.

Para determinação dos parâmetros ótimos de furação foi utilizado o Método Taguchi. O mesmo caracteriza-se pela necessidade de um menor número de experimentos, buscando minimizar o tempo e o custo dos processos.

\subsection{Método Taguchi}

O método Taguchi foi desenvolvido pelo engenheiro e estatístico Genichi Taguchi e tem como objetivos o controle da qualidade de produtos e processos, bem como o aumento da produtividade e a redução de custos. Montgomery (2004) descreve que um processo transforma um material de entrada em um produto de saída, sendo que este último pode ter uma ou mais respostas. No processo, há variáveis que são controláveis e outras nãocontroláveis, as quais podem ser chamadas de fatores de ruído. A figura 2 mostra o modelo geral de um processo.

\section{Fatores de entrada}

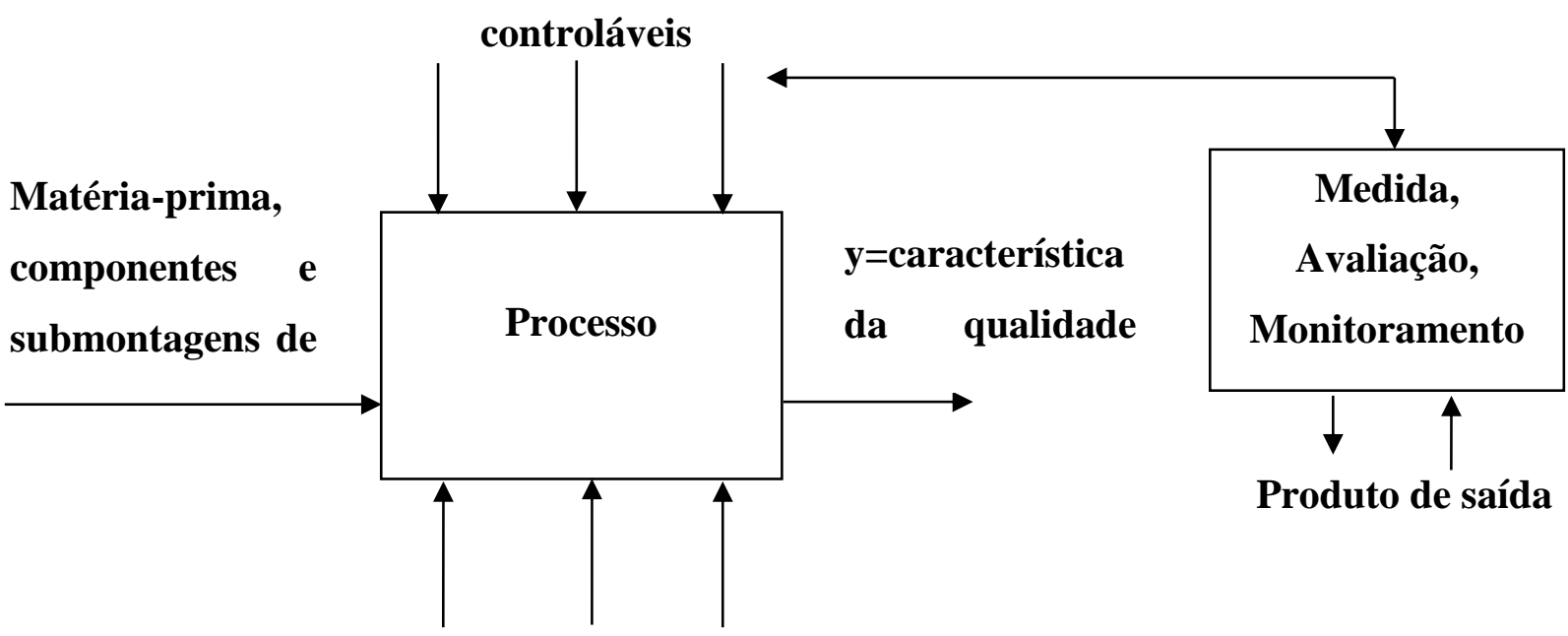

\section{Fatores de entrada}

não-controláveis

Figura 2 - Entradas e saídas de um processo de produção Fonte - Montgomery (2004, p. 9). 


\subsection{Seleção do arranjo ortogonal (AO)}

Para a seleção do arranjo ortogonal adequado, o qual representa o $\mathrm{n}^{\circ}$ de experimentos a serem realizados, utiliza-se como base a tabela 1 . O arranjo ortogonal a ser escolhido depende do número de fatores e do número de níveis de cada fator.

Tabela 1 - Arranjos ortogonais

\begin{tabular}{|c|c|c|c|c|c|c|}
\hline \multirow{2}{*}{$\begin{array}{c}\text { Arranjo } \\
\text { Ortogonal }\end{array}$} & \multirow{2}{*}{$\begin{array}{l}N^{o} \text { de } \\
\text { linhas }\end{array}$} & \multirow{2}{*}{$\begin{array}{c}N^{0} \text { máximo } \\
\text { de fatores }\end{array}$} & \multicolumn{4}{|c|}{$\mathrm{N}^{0}$ máximo de fatores com esses níveis } \\
\hline & & & 2 & 3 & 4 & 5 \\
\hline$L_{4}$ & 4 & 3 & 3 & - & - & - \\
\hline$L_{8}$ & 8 & 7 & 7 & - & - & - \\
\hline$L_{9}$ & 9 & 4 & - & 4 & - & - \\
\hline$L_{12}$ & 12 & 11 & 11 & - & - & - \\
\hline$L_{16}$ & 16 & 15 & 15 & - & - & - \\
\hline$L^{\prime}{ }_{16}$ & 16 & 5 & - & - & 5 & - \\
\hline$L_{18}$ & 18 & 8 & 1 & 7 & - & - \\
\hline$L_{25}$ & 25 & 6 & - & - & - & 6 \\
\hline$L_{27}$ & 27 & 13 & - & 13 & - & - \\
\hline$L_{32}$ & 32 & 31 & 31 & - & - & - \\
\hline$L_{32}^{\prime}$ & 32 & 10 & 1 & - & 9 & - \\
\hline$L_{36}$ & 36 & 23 & 11 & 12 & - & - \\
\hline$L_{36}^{\prime}$ & 36 & 16 & 3 & 13 & - & - \\
\hline$L_{50}$ & 50 & 12 & 1 & - & - & 11 \\
\hline$L_{54}$ & 54 & 26 & 1 & 25 & - & - \\
\hline$L_{64}$ & 64 & 63 & 63 & - & - & - \\
\hline$L_{64}^{\prime}$ & 64 & 21 & - & - & 21 & - \\
\hline$L_{81}$ & 81 & 40 & - & 40 & - & - \\
\hline
\end{tabular}

Fonte: Phadke (1989, p. 152).

\section{3 Índice Sinal-Ruído}

De acordo com a quantidade de variações que aparece como resposta, os fatores de controle que contribuem para a redução de variação da qualidade podem ser identificados. A 
relação sinal-ruído combina repetições em um valor que demonstra o quanto de variação existe (ROSS, 1991).

Para a determinação do nível ótimo da característica e, assim, atender as especificações do produto ou atingir a robustez de um processo, o método Taguchi utiliza a relação sinal-ruído ( $\mathrm{S} / \mathrm{N}$ ou $\eta$ ), que apresenta três condições (Equações 1, 2 e 3), de acordo com Phadke (1989):

\section{Menor o melhor:}

$$
\eta=-10 \log _{10}\left[\frac{1}{n} \sum_{i=1}^{n} y_{i}^{2}\right]
$$

Maior o melhor:

$$
\eta=-10 \log _{10}\left[\frac{1}{n} \sum_{i=1}^{n} \frac{1}{y_{i}^{2}}\right]
$$

\section{Nominal o melhor:}

$$
\eta=10 \log _{10} \frac{\mu^{2}}{\sigma^{2}}
$$

Sendo que, para a Equação (3), tem-se as Equações (4) e (5):

$$
\begin{gathered}
\mu=\frac{1}{n} \sum_{i=1}^{n} y_{i} \\
\sigma^{2}=\frac{1}{n-1} \sum_{i=1}^{n}\left(y_{i}-\mu\right)^{2}
\end{gathered}
$$

E $n, i, y$ e $\mu$ denotam o número de repetições das observações, índice, valor observado e valor nominal, respectivamente.

Minimizar a perda da qualidade significa maximizar a razão sinal-ruído, ou seja, o nível que apresentar uma maior S/N será considerado o nível ótimo.

\section{METODOLOGIA}

\subsection{Corpo de prova e condições experimentais}

ForSci.: r. cient. IFMG, Formiga, v. 6, n. 2, e00402, out. 2018, Edição Especial. 
Foram utilizados corpos de prova do material sanduíche com dimensões de 130mmx 100mmx4mm. As propriedades mecânicas do material sanduíche utilizado estão relacionadas na tabela 2 . Sobre cada corpo de prova foram realizados furos, variando-se diversos parâmetros, conforme a tabela 3 .

Tabela 2 - Propriedades Mecânicas do material sanduíche

\begin{tabular}{cccc}
\hline Material & $\begin{array}{c}\text { Módulo de } \\
\text { Elasticidade (GPa) }\end{array}$ & $\begin{array}{c}\text { Resistência à } \\
\text { tração (MPa) }\end{array}$ & $\begin{array}{c}\text { Alongamento } \\
(\mathbf{\%})\end{array}$ \\
\hline ACM-4mm & $6,51 \pm 1,28$ & $30,31 \pm 1,78$ & $4 \pm 1,4$ \\
\hline
\end{tabular}

Tabela 3 - Condições experimentais

\begin{tabular}{|c|c|c|c|c|}
\hline \multirow{2}{*}{ Símbolo } & \multirow{2}{*}{ Parâmetros } & \multicolumn{3}{|c|}{ Níveis } \\
\hline & & 1 & 2 & 3 \\
\hline A & $\begin{array}{l}\text { Velocidade } \\
\text { de corte }- \\
\text { Vc }(\mathrm{m} / \mathrm{min})\end{array}$ & 24 & 48 & 72 \\
\hline B & $\begin{array}{c}\text { Avanço }-f \\
(\mathrm{~mm} / \mathrm{rev})\end{array}$ & 0,05 & 0,15 & 0,25 \\
\hline $\mathrm{C}$ & Ferramenta & $\begin{array}{c}\text { Sem } \\
\text { revestimento }\end{array}$ & $\begin{array}{c}\text { Com } \\
\text { revestimento } \\
\text { de } \mathrm{TiO}_{2}\end{array}$ & $\begin{array}{c}\text { Sem } \\
\text { revestimento } \\
\text { e com pré- } \\
\text { furo }\end{array}$ \\
\hline
\end{tabular}

\subsection{Centro de usinagem e sistema de aquisição de dados}

O centro de usinagem ROMI, modelo Discovery 560, com potência máxima de 9000 W e rotação máxima de $7500 \mathrm{rpm}$, foi utilizado para a realização dos experimentos. $\mathrm{O}$ deslocamento máximo nos eixos $\mathrm{X}=560 \mathrm{~mm}, \mathrm{Y}=406 \mathrm{~mm}$ e $\mathrm{Z}=508 \mathrm{~mm}$. Mínimo incremento de posicionamento: 0,001 $\mathrm{mm}$ e $0,001^{\circ}$.

$\mathrm{Na}$ figura 3, tem-se a montagem do sistema de aquisição de dados, bem como o dinamômetro extensométrico, amplificador, placa de aquisição de dados, fontes de 5 e 12 volts e o computador com software LabVIEW. A ferramenta utilizada foi uma broca helicoidal de diâmetro $5 \mathrm{~mm}$ e para o pré-furo uma broca de $3 \mathrm{~mm}$. 


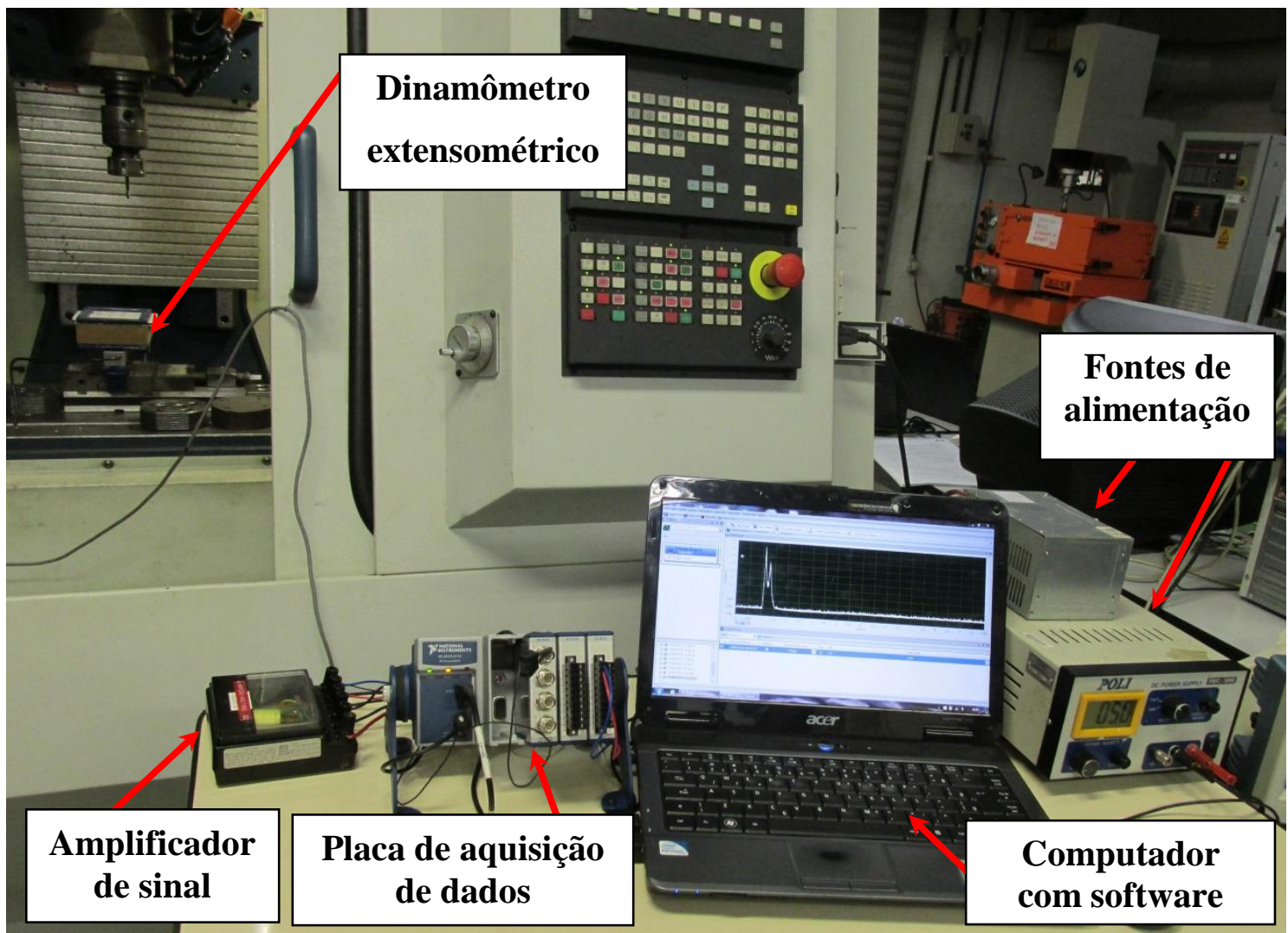

Figura 3 - Montagem do sistema da aquisição de dados

Para a medição da força de avanço durante a furação do material sanduíche, foi usado um dinamômetro extensométrico com célula de carga de $10 \operatorname{Kgf}(98,0665$ N).

Para a medição das rebarbas do furo, utilizou-se microscópio Askania, modelo GSZ 2T Germany, com um micrômetro de resolução de $0,01 \mathrm{~mm}$ e para a medição do diâmetro máximo dos furos foi utilizada a máquina de medição por coordenadas TESA, modelo MICRO HITE 3D, com o software REFLEX SCAN cuja resolução é 0,001 mm.

De forma a minimizar o número de experimentos para determinação das condições ótimas de corte, foi utilizado o Método Taguchi, com um arranjo L9, sendo que o mesmo foi determinado por meio do software Minitab 17.

\section{RESULTADOS E DISCUSSÃO}

Os resultados obtidos durante a operação de furação para a força de avanço $\left(\mathrm{F}_{\mathrm{f}}\right)$, altura da rebarba $(\mathrm{h})$ e diâmetro máximo $\left(\mathrm{D}_{\text {máx }}\right)$ constam na tabela 4. 
Tabela 4 - Arranjo L9 e resultados médios da força de avanço, altura da rebarba e diâmetro máximo

\begin{tabular}{ccccccc}
\hline Experimento & Ferramenta & $\begin{array}{c}\text { Vc } \\
(\mathbf{m} / \mathbf{m i n})\end{array}$ & $\begin{array}{c}\boldsymbol{f} \\
(\mathbf{m m} / \mathbf{r e v})\end{array}$ & $\mathbf{F}_{\mathbf{f}}(\mathbf{N})$ & $\mathbf{h}(\mathbf{m m})$ & $\mathbf{D}_{\text {máx }}(\mathbf{m m})$ \\
\hline 1 & Sem Rev. & 24 & 0,05 & 57,21 & 0,61 & 5,034 \\
2 & Sem Rev. & 48 & 0,15 & 78,38 & 0,74 & 5,135 \\
3 & Sem Rev. & 72 & 0,25 & 75,43 & 0,97 & 5,050 \\
4 & Com Rev. & 24 & 0,15 & 85,67 & 0,85 & 5,036 \\
5 & Com Rev. & 48 & 0,25 & 84,65 & 0,84 & 5,063 \\
6 & Com Rev. & 72 & 0,05 & 52,48 & 0,36 & 5,098 \\
7 & Pré-furo & 24 & 0,25 & 68,09 & 1,44 & 4,999 \\
8 & Pré-furo & 48 & 0,05 & 40,01 & 1,78 & 5,057 \\
9 & Pré-furo & 72 & 0,15 & 46,84 & 1,60 & 5,037 \\
\hline
\end{tabular}

\subsection{Força de Avanço}

A tabela 5 mostra o nível de influência de cada parâmetro utilizado na força de avanço.

Tabela 5 - Tabela resposta da razão sinal-ruído menor é o melhor para a força de avanço

\begin{tabular}{cccc}
\hline Nível & $\begin{array}{c}\text { Ferramenta } \\
(\mathbf{d B})\end{array}$ & $\begin{array}{c}\text { Velocidade } \\
\text { de corte } \\
(\mathbf{d B})\end{array}$ & $\begin{array}{c}\text { Avanço } \\
(\mathbf{d B})\end{array}$ \\
\hline 1 & $-36,86$ & $-36,82$ & $-33,86$ \\
2 & $-37,20$ & $-36,16$ & $-36,65$ \\
3 & $-34,04$ & $-35,12$ & $-37,59$ \\
Delta & 3,16 & 1,70 & 3,72 \\
Influência & 2 & 3 & 1 \\
\hline
\end{tabular}

De acordo com a tabela 5, a ordem de influência dos fatores na força de avanço é a seguinte: avanço, ferramenta e velocidade de corte. A figura 4 mostra a razão sinal-ruído para a força de avanço. 


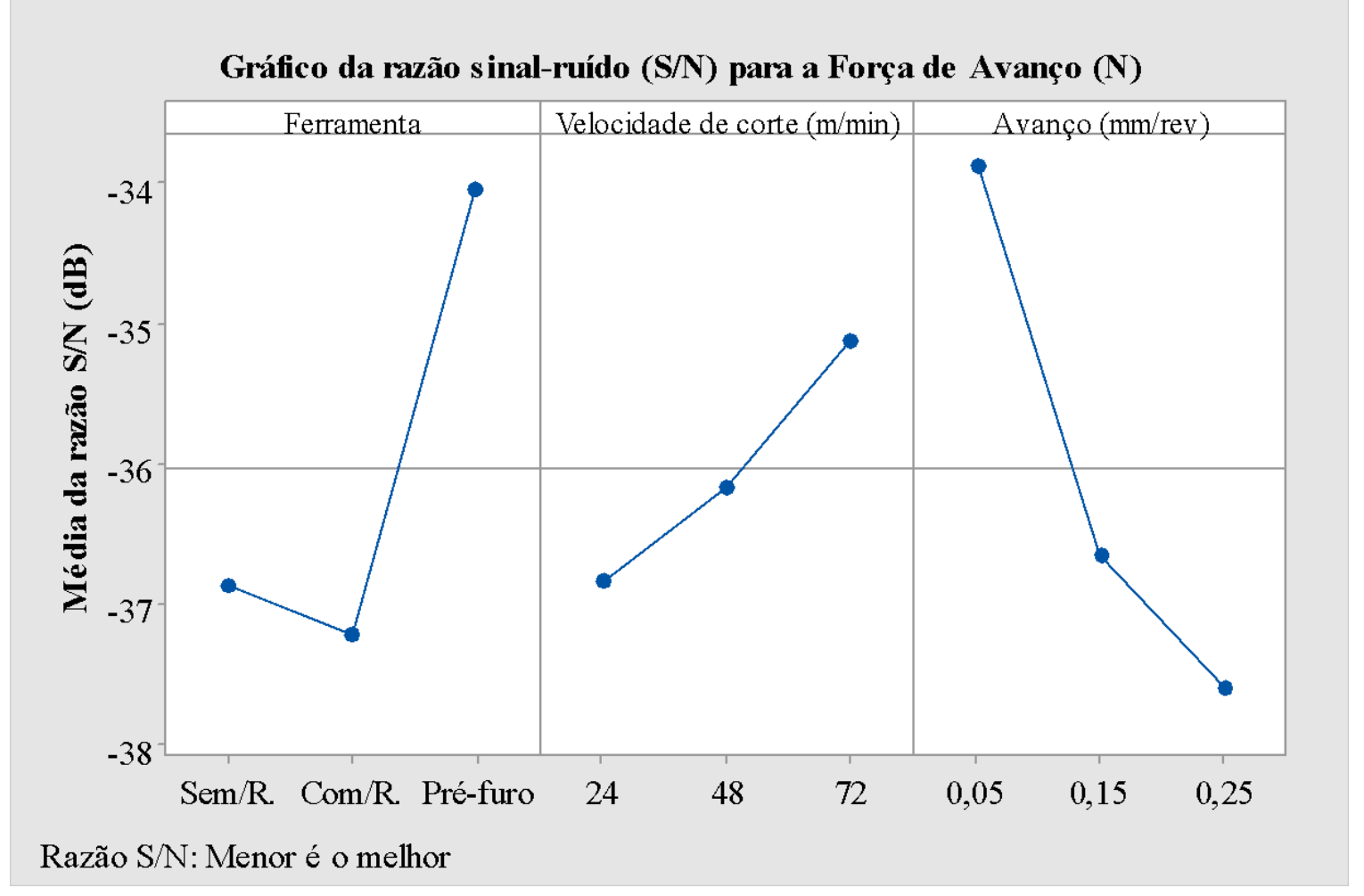

Figura 4 - Gráfico da razão sinal-ruído menor é o melhor para a força de avanço

Os parâmetros ótimos para a furação, tendo como objetivo menor força de avanço, são:

- $\quad$ Ferramenta: pré-furo;

- Velocidade de corte: $72 \mathrm{~m} / \mathrm{min}$;

- Avanço: $0,05 \mathrm{~mm} / \mathrm{rev}$.

Com a utilização do pré-furo, durante a furação com a broca de $5 \mathrm{~mm}$ há a retirada de uma menor quantidade de material, portanto a força de avanço é menor. A peça de trabalho oferece menor resistência ao corte com a utilização de uma velocidade de corte de $72 \mathrm{~m} / \mathrm{min}$ e com relação ao avanço, como o mesmo é diretamente proporcional à força de avanço, tem-se menor força $\operatorname{com} f=0,05 \mathrm{~mm} / \mathrm{rev}$.

\subsection{Altura da Rebarba}


A tabela 6 mostra o nível de influência de cada parâmetro utilizado na altura da rebarba. De acordo com a mesma, a ordem de influência dos fatores na altura da rebarba é a seguinte: ferramenta, avanço e velocidade de corte.

Tabela 6 - Tabela resposta da razão sinal-ruído menor é o melhor para a altura da rebarba

\begin{tabular}{cccc}
\hline Nível & $\begin{array}{c}\text { Ferramenta } \\
(\mathbf{d B})\end{array}$ & $\begin{array}{c}\text { Velocidade } \\
\text { de corte } \\
(\mathbf{d B})\end{array}$ & $\begin{array}{c}\text { Avanço } \\
(\mathbf{d B})\end{array}$ \\
\hline 1 & 2,39111 & 0,84592 & 2,71965 \\
2 & 3,93333 & $-0,29287$ & $-0,01847$ \\
3 & $-4,08602$ & 1,68537 & $-0,46276$ \\
Delta & 8,01935 & 1,97825 & 3,18241 \\
Influência & 1 & 3 & 2 \\
\hline
\end{tabular}

A figura 5 mostra a razão sinal-ruído para a Altura da Rebarba, e, de acordo com a mesma, os parâmetros ótimos para a furação, tendo como objetivo menor altura da rebarba, são:

- Ferramenta: com revestimento;

- $\quad$ Velocidade de corte: $72 \mathrm{~m} / \mathrm{min}$;

- Avanço: $0,05 \mathrm{~mm} / \mathrm{rev}$.

$\mathrm{O}$ revestimento de $\mathrm{TiO}_{2}$ contribuiu para um menor coeficiente de atrito entre e a peça e a ferramenta, facilitando o cisalhamento. Para a velocidade de corte de $72 \mathrm{~m} / \mathrm{min}$ o cisalhamento também é facilitado, visto que o aumento de temperatura ocasiona uma menor resistência da peça. Já com relação ao avanço de $0,05 \mathrm{~mm} / \mathrm{rev}$, a quantidade de material retirado por volta é menor. Todos esses fatores ocasionam uma diminuição na altura da rebarba. 


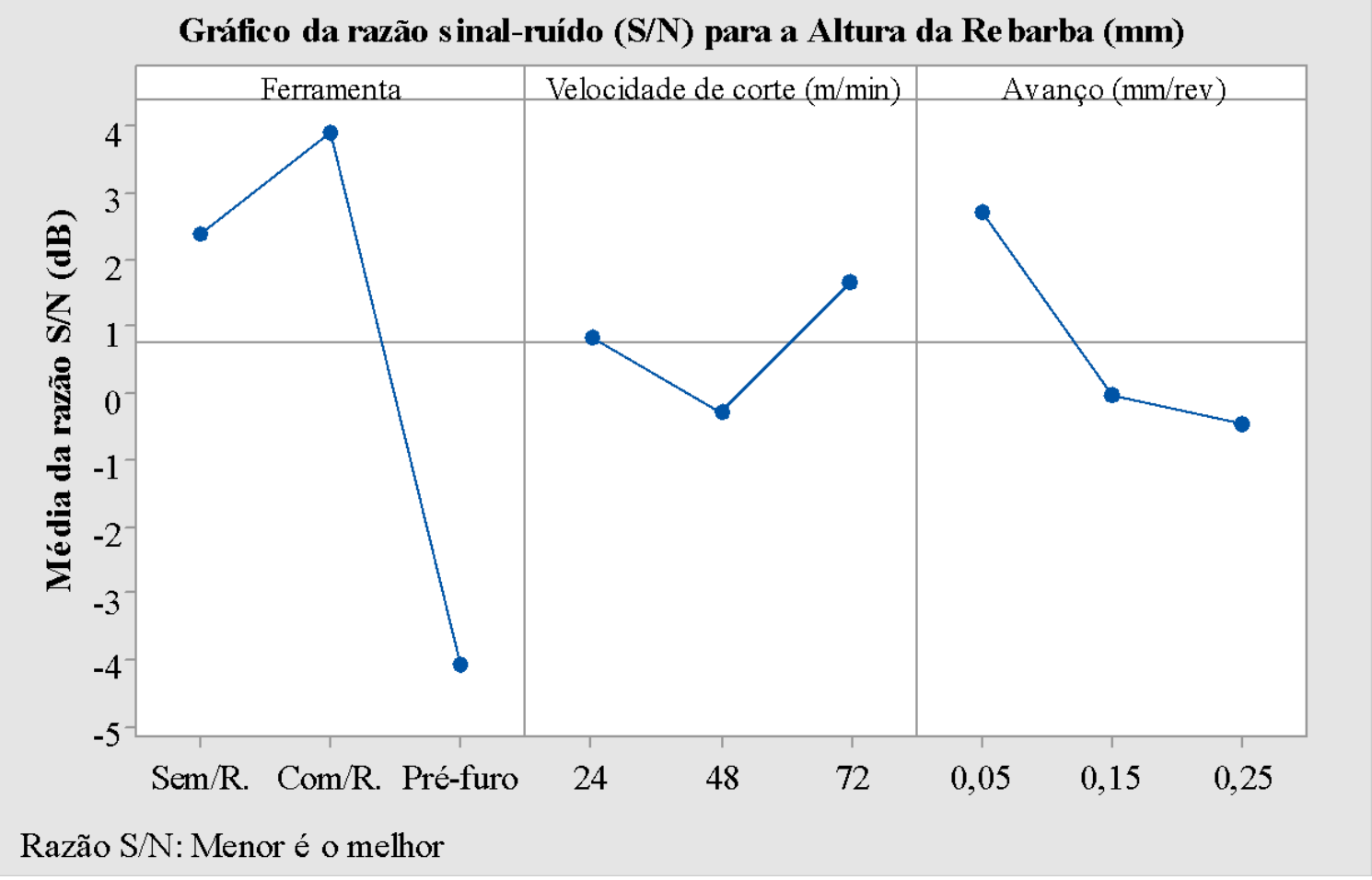

Figura 5 - Gráfico da razão sinal-ruído menor é o melhor para a altura da rebarba

\subsection{Diâmetro Máximo}

A tabela 7 mostra o nível de influência de cada parâmetro utilizado no diâmetro máximo.

Tabela 7 - Tabela resposta da razão sinal-ruído menor é o melhor para o diâmetro máximo

\begin{tabular}{cccc}
\hline Nível & $\begin{array}{c}\text { Ferramenta } \\
(\mathbf{d B})\end{array}$ & $\begin{array}{c}\text { Velocidade } \\
\text { de corte } \\
(\mathbf{d B})\end{array}$ & $\begin{array}{c}\text { Avanço } \\
(\mathbf{d B})\end{array}$ \\
\hline 1 & $-14,10$ & $-14,02$ & $-14,09$ \\
2 & $-14,09$ & $-14,13$ & $-14,10$ \\
3 & $-14,03$ & $-14,09$ & $-14,04$ \\
Delta & 0,07 & 0,11 & 0,05 \\
Influência & 2 & 1 & 3
\end{tabular}

De acordo com a tabela 7, a ordem de influência dos fatores no diâmetro máximo é a seguinte: velocidade de corte, ferramenta e avanço. A figura 5 mostra a razão sinal-ruído para o Diâmetro Máximo. 


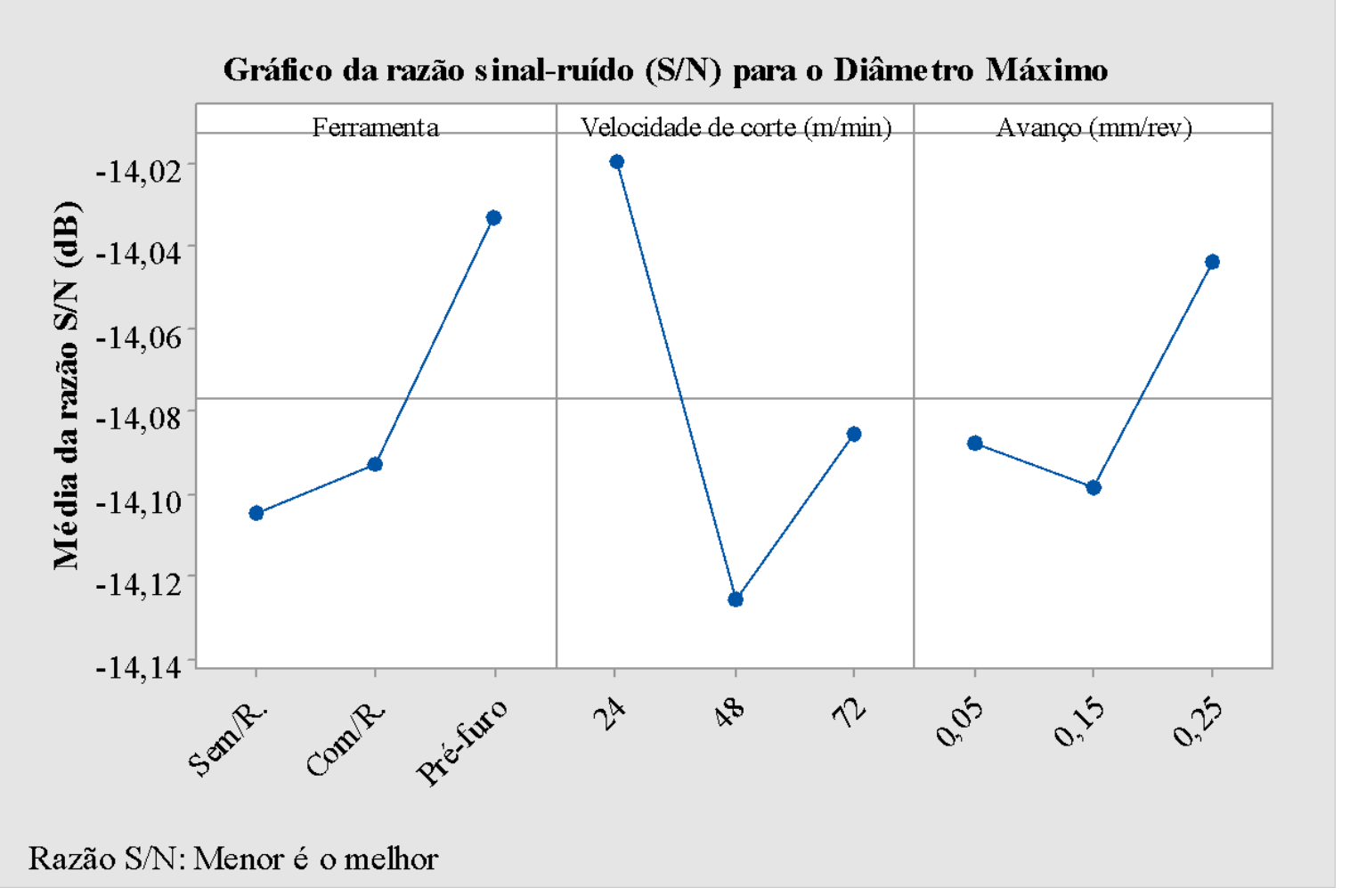

Figura 6 - Gráfico da razão sinal-ruído menor é o melhor para o diâmetro máximo

Os parâmetros ótimos para a furação, tendo como objetivo menor diâmetro máximo, são:

- $\quad$ Ferramenta: pré-furo;

- Velocidade de corte: $24 \mathrm{~m} / \mathrm{min}$;

- $\quad$ Avanço: $0,25 \mathrm{~mm} / \mathrm{rev}$.

Com a utilização de pré-furo há menores níveis de vibração, visto que as forças também são menores, o que contribui para menores variações de diâmetro em torno do valor de $5 \mathrm{~mm}$. Também contribuem para menores variações de diâmetro, a menor velocidade de corte $(24 \mathrm{~m} / \mathrm{min})$, uma vez que a temperatura obtida é mais baixa, e o avanço de $0,25 \mathrm{~mm}$ /rev, pois a ferramenta permanece na peça por menor tempo em comparação aos outros avanços.

As condições ótimas obtidas para a furação do PEALL, tendo como objetivo menor é o melhor, para a força de avanço, altura da rebarba e diâmetro máximo por meio do Método Taguchi foram obtidas. 


\title{
4 CONCLUSÕES
}

Por meio do método Taguchi foi possível a determinação dos parâmetros ótimos de corte para a furação do material sanduíche com um arranjo reduzido de experimentos, diminuindo-se, assim, custos e tempo. Para a força de avanço, a melhor combinação é utilizando pré-furo, velocidade de corte de $72 \mathrm{~m} / \mathrm{min}$ e avanço de $0,05 \mathrm{~mm} / \mathrm{rev}$.

Com relação à altura da rebarba, a melhor combinação foi ferramenta com revestimento, velocidade de corte de $72 \mathrm{~m} / \mathrm{min}$ e avanço $0,05 \mathrm{~mm} / \mathrm{rev}$. Por fim, para obtenção de diâmetro com menor variação, os melhores parâmetros foram pré-furo, velocidade de corte de $24 \mathrm{~m} / \mathrm{min}$ e avanço de $0,25 \mathrm{~mm} / \mathrm{rev}$. A melhor combinação de parâmetros a ser utilizada depende do objetivo que se deseja alcançar.

\section{AGRADECIMENTOS}

Os autores dessa pesquisa agradecem aos departamentos de Engenharia de Produção e Engenharia Mecânica da Universidade Federal de Minas Gerais pela disponibilização do Laboratório de Usinagem e Automação. Agradecem às agências de fomento Capes, CNPQ e Fapemig e ao Instituto Federal de Minas Gerais (IFMG) - Campus Bambuí pela oportunidade de divulgação do trabalho.

\section{APPLICATION OF THE TAGUCHI METHOD IN THE OPTIMIZATION OF THE PARAMETERS OF THE DRILLING OPERATION OF A SANDWICH MATERIAL}

\begin{abstract}
Concomitantly with the need to develop materials with superior qualities arises the need to process these new materials, presenting characteristics and / or difficulties for the manufacture of components. In this sense, the composite materials have several characteristics, such as low weight, rigidity and high resistance, which allow the use in several areas of modern industry. Sandwich materials are a type of structural composite and have basically two faces and a core. In order to achieve products with the desired qualities, different manufacturing processes are used, among them machining. The drilling operation is one of the most used for the joining of materials. A number of problems may occur, such as delamination, circularity error and burrs. In this sense, the present work has the objective of determining the optimal cutting conditions, by means of the Taguchi Method, for the drilling of a sandwich type material, made of aluminum and polyethylene. Three types of tools were used, three cutting speeds and three feeds. The optimum cutting conditions obtained were established considering the lower feed force, lower burr height and lower maximum diameter.
\end{abstract}

Keywords: Drilling. Sandwich material. Taguchi Method. 


\section{REFERÊNCIAS}

DINIZ, A. E.; MARCONDES, F. C.; COPPINI, N. L. Tecnologia da usinagem dos

materiais. 5. ed. São Paulo: Artliber, 2006.

KHORAN, M. et al. Investigation of drilling composite sandwich structures. The

International Journal of Advanced Manufacturing Technology, London, v. 76, n. 9-12, p. 1927-1936, fev. 2015. Disponível em: <https://link.springer.com/article/10.1007/s00170-0146427-x.>. Acesso em: 22 dez. 2017.

MACHADO, Á. R. et al. Teoria da usinagem dos materiais. 3. ed. São Paulo: Blucher, 2015.

MONTGOMERY, D. C. Introdução ao controle estatístico da qualidade. 4. ed. Rio de Janeiro: LTC, 2004.

OLIVEIRA, V. V. de. Influência da geometria de brocas na furação do ferro fundido vermicular. 2008. 173 f. Dissertação (Mestrado em Engenharia Mecânica e de Materiais) Universidade Federal Tecnológica do Paraná, Curitiba, 2008.

PHADKE, M. S. Quality engineering using robust design. USA: Prentice-Hall, 1989.

PILNÝ, L. et al. Hole quality and burr reduction in drilling aluminium sheets. CIRP Journal of Manufacturing Science and Technology, Netherlands, v. 5, n. 2, p. 102-107, 2012. Disponível em: <https://www.sciencedirect.com/science/article/pii/S175558171200020X>. Acesso em: 22 dez. 2017.

ROSS, P. J. Aplicações das técnicas Taguchi na engenharia da qualidade. São Paulo: McGraw-Hill, 1991.

STEMMER, E.C. Ferramentas de corte. 6. ed. Florianópolis: UFSC, 2005.

ZITOUNE, R.; KRISHNARAJ, V.; COLLOMBET, F. Study of drilling of composite material and aluminium stack. Composite Structures, Oxford, v. 92, n. 5, p. 1246-1255, abr. 2010. Disponível em: <https://www.sciencedirect.com/science/article/pii/S0263822309004188>. Acesso em: 22 dez. 2017.

\section{DADOS DOS AUTORES:}

\section{Bruna Aparecida Rezende:}

Lattes: http://lattes.cnpq.br/6085282319322315

E-mail: bruna.rezende@ifmg.edu.br

Possui graduação em Engenharia de Produção pelo Centro Universitário de Formiga (2012). Pós-graduada em Engenharia de Segurança do Trabalho pela PUC Minas (2015). Mestre em 
Engenharia Mecânica na área Processos de Fabricação pela UFMG (2016). Atualmente é doutoranda em Engenharia de Produção pela UFMG na área de processos de fabricação e materiais e professora EBTT no IFMG-Campus Bambuí.

\section{Michele Lisboa Silveira}

Lattes: http://lattes.cnpq.br/0123998424653189

E-mail: michelesilveira1991@ gmail.com

Doutoranda no curso em Engenharia de Produção na Universidade Federal de Minas Gerais, onde participa do grupo de pesquisa do Laboratório de Usinagem e Automação. Possui mestrado em Engenharia de Produção na Universidade Federal de Minas Gerais e Graduação em Engenheira de Produção pela Universidade Federal de Ouro Preto. Atuou como professora substituta na Universidade Federal de Ouro Preto. Experiência na área de Engenharia de Produção, com ênfase em gerenciamento de projetos e planejamento e controle da produção. Atualmente leciona no curso de Engenharia de Produção da Faculdade Alis em Itabirito - MG.

\section{Juan Carlos Campos Rubio}

Lattes: http://lattes.cnpq.br/9138858373933921

E-mail: juan@ufmg.br

Possui graduação em Engenharia Mecânica pela Universidade Federal de Santa Maria (1988), mestrado em Engenharia Mecânica pela Universidade Federal de Santa Catarina (1992) e doutorado em Engenharia Mecânica pela Universidade de São Paulo (2000). Atualmente é professor associado da Universidade Federal de Minas Gerais, e faz parte do corpo editorial da International Journal of Mechatronics and Manufacturing Systems (IJMMS), do Journal of Modern Manufacturing Technology (JMMT) e do International Journal of Composite Materials. Tem experiência na área de Engenharia Mecânica e de Produção, com ênfase em Processos de Fabricação e Automação, atuando principalmente nos seguintes temas: engenharia de precisão, projeto, automação, usinagem e máquinas-ferramenta CNC. 